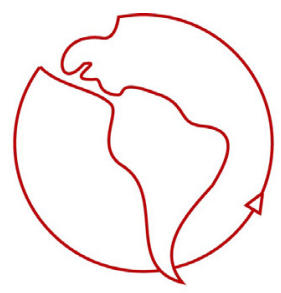

\title{
Globalización, Capital Humano y Alfabetización Digital
}

Dr. Carlos Haefner Velásquez

Universidad de Chile y Universidad Austral de Chile

chaefner@uchile.cl

\section{Globalización y Brechas Sociales}

Existe una relación virtuosa entre el fortalecimiento de las capacidades humanas y el desempeño económico. Por tanto, es fácil concluir que en un mundo que se globaliza el motor de la dinámica del desarrollo lo constituye el capital humano.

La observación de diversas experiencias de países exitosos tales como Corea, Finlandia, Portugal, Nueva Zelanda en cuanto a desarrollo e innovación permite concluir que ello fue posible gracias a la generación de políticas educativas de amplia cobertura, calidad y adaptación dinámica un mundo cada vez más complejo y cambiante. Y, por cierto, en una apuesta por la formación y capacitación permanente de sus fuerzas de trabajo; entendiendo que tal formación sea orientada a lograr trabajadores cuya principal cualidad sea la flexibilidad frente a los nuevos conocimientos y formas de gestión; como también su capacidad de aprendizaje continúo y de adaptación a los permanentes cambios de orden tecnológico y organizacional. Todo ello acompañado de una fuerte innovación tecnológica, fundado en políticas de inversión de largo plazo que han sido posibles en la en la medida que el gobierno, las universidades y empresa privada se constituyeron en socios estratégicos para la formación de capital humano avanzado y con ello potenciar en forma espectacular investigación y desarrollo tecnológico de punta.

Recientemente los profesores J.J Brunner y G. Elacqua de la Universidad Adolfo Ibáñez han dado a conocer un estudio referido al Capital Humano en Chile (2003). En este interesante trabajo se hace un descarnado análisis de nuestras fortalezas y debilidades que tenemos como país en esta materia. Una de las conclusiones del informe nos señala que Chile cuenta con un moderado "stock" de capital humano cuya acumulación ha sido más bien lenta, cuya distribución es altamente desigual, y que su renovación es escasa y su calidad y desempeño resultan inadecuados para enfrentar los requerimientos de una globalización altamente asimétrica y desigual.

Como afirma el ganador del premio Nobel de economía Amarya Sen, el capitalismo global está más preocupado por extender su dominio de las relaciones comerciales que, por ejemplo, establecer la democracia, universalizar la educación básica o mejorar las oportunidades sociales de los más pobres. En este sentido, la pregunta que debemos hacernos radica en torno a que tan eficientes son los arreglos institucionales que tienen los países periféricos para vencer las barreras del desarrollo que se imponen en el actual modelo económico - social que se mundializa.

La cuestión central de la discusión que debemos desarrollar hoy no es la globalización en sí, ni tampoco el uso de los mercados como institución, sino la falta de equidad en el balance de los arreglos institucionales, especialmente los educacionales, lo que provoca una distribución muy desigual de los beneficios de la globalización.

\section{Capital Humano en Chile: Desafíos Pendientes}

Nuestro país ha experimentado en los últimos 25 años un fuerte proceso de cambio de su matriz sociocultural, gatillado por diversos procesos modernizadores. Es el caso del sistema educativo. De 
acuerdo a las cifras del último CENSO, la escolaridad promedio alcanzó a 8,5 años en 2002, comparado con 7,5 años una década antes. En tanto, entre la fuerza laboral este indicador llegó a 10,5 años, en contraste con 9,2 años en 1992. La obligatoriedad de la educación secundaria se viene a sumar a estos logros, en la medida que su cumplimiento permitirá al país comenzar a soñar con el umbral del desarrollo. Sin embargo, el cambio más significativo durante los últimos años se observa en la educación superior. Casi un tercio de quienes egresan de la enseñanza media continúan su proceso educativo y, desde 1992, el número de profesionales y técnicos en Chile se ha más que duplicado, alcanzando al 16,4\% de la población en el año 2002.

Estos logros son muy interesantes, pero no suficientes; especialmente para cubrir las exigencias que se derivan de un mundo global. El desafío sustantivo que tiene hoy es evaluar como lo esta haciendo en lo que respecta a su inserción en la sociedad del conocimiento. Teniendo a la vista los resultados que el país ha obtenido en algunas evaluaciones internacionales en donde se le compara con otros países, tanto desarrollados como emergentes, los resultados son preocupantes. Por ejemplo, los resultados de la última medición (2003) en la que hemos participado como país a nivel internacional el "Programa para la Evaluación Internacional de Estudiantes" (PISA, por sus siglas en inglés) a cargo de la Organización para la Cooperación y el Desarrollo Económico (OCDE), y que tiene como objetivo medir los conocimientos escolares en lectura, matemáticas y ciencias; nuestro país ocupo el lugar 36 entre 41 países evaluados.

Tales resultados y otros que se conocen públicamente, invitan, por lo menos, a una discusión abierta y sincera en torno al diseño, aplicación y evaluación de nuestras políticas educacionales. Para que ello ocurra debemos primeramente desectorializar la discusión y volcar el análisis a una mirada global del sistema educativo nacional enmarcado en el contexto de una sociedad globalizada que avanza asimétricamente y va dejando una importante estela de excluidos de los frutos del desarrollo. Vale decir, debemos asumir que estamos en un escenario que se caracteriza por un crecimiento exponencial del conocimiento $\mathrm{y}$, en consecuencia, por la distinción de países ganadores y perdedores en la carrera por disminuir la brecha digital y educativa. El sólo dato que en Chile apenas el 1\% de los estudiantes logró el grado más alto de lectura, el $28 \%$ el mínimo, y $20 \%$ no entiende lo que lee, nos indica que debemos superar los análisis parciales que muchas veces son periféricos al núcleo real de explicación de nuestras desventajas competitivas en esta materia.

De hecho, para la OCDE la explicación de estos resultados debemos encontrarlos en diversas variables, entre ellas el factor del presupuesto educativo y la desigualdad social. Vale decir, cuando el gasto por alumno en las instituciones educativas aumentan, también se incrementa el desempeño medio del país. A estos factores, se deben tener en consideración que el entorno del hogar influye en el éxito educativo y el status socioeconómico puede reforzar sus efectos. La llave del aprendizaje -se sostiene- es que las escuelas no ayuden a reproducir patrones existentes de privilegio, sino más bien den iguales oportunidades a los jóvenes; que la escuela aproveche todos los recursos a su alcance, que los docentes estén especializados y comprometidos con su tarea y que los planteles tengan cada vez mayor autonomía.

En la misma dirección, si nos comparamos, por ejemplo, con otros países con economías emergentes y competitivas las diferencias de logro en la variable capital humano avanzado es altamente preocupante, pues tenemos un rezago importante en lo que respecta a Profesionales y técnicos. Estudios recientes indican que en relación a la fuerza de trabajo, el stock de profesionales y técnicos apenas supera el $10 \%$. 


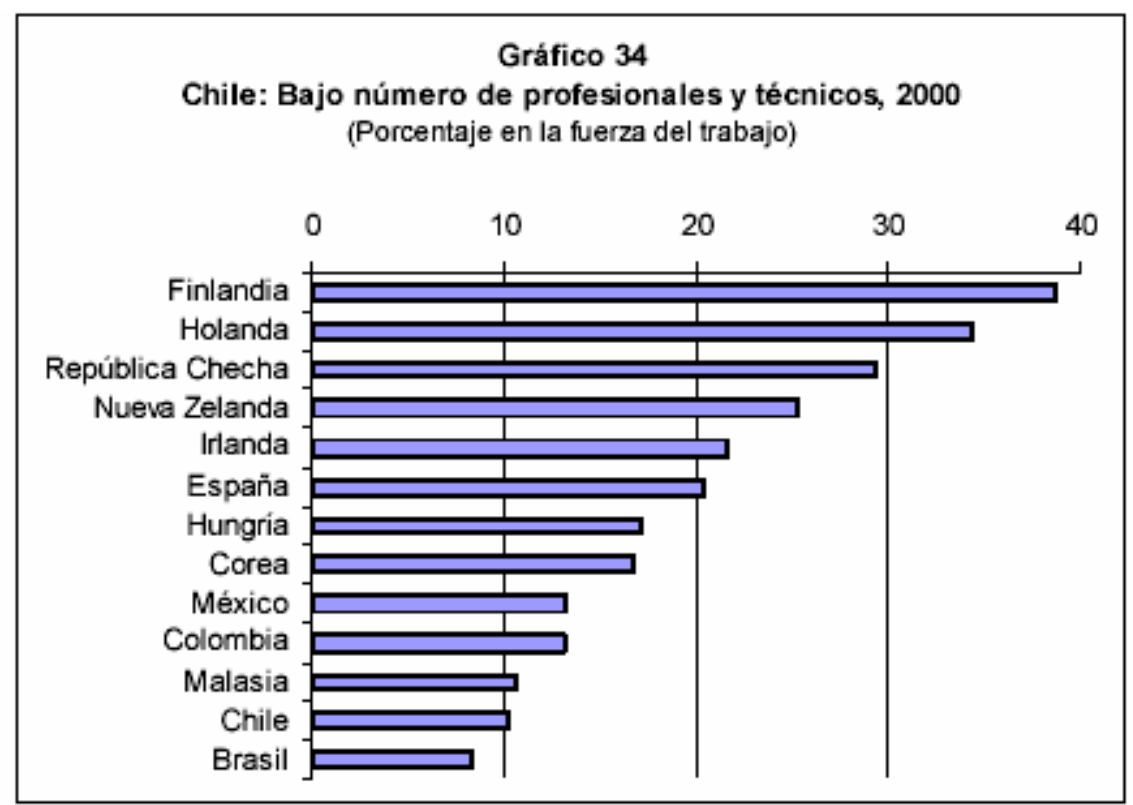

Tomado de Brunner y Elaqua (2003), según Base de datos del Banco Mundial.

Esta regresividad la encontramos también en la distribución regional; no sorprende que en esta materia haya una alta concentración de profesionales y técnicos en la Región Metropolitana (50,5\% del total nacional). En cuanto a la distribución regional, se advierte una concentración de profesionales y técnicos aún mayor que la de la población en la R. Metropolitana y un desbalance negativo en las regiones IV, VI, VII y X.

Por otra parte, según las cifras entregadas por CONICYT (2004), nuestro país muestra avances en lo referido a formación de doctorados, por ejemplo en 1999 se graduaban alrededor de 50 doctores, lográndose que para el año 2003 se graduaron en Chile alrededor de 160 doctores y este año existen alrededor de 500 estudiantes de doctorados. Se estima que hacía el 2008 se graduarán en Chile unos 400 doctores y una centena en el extranjero. Hay que señalar, sin embargo, que la cifra óptima que se plantea para lograr indicadores importantes de competitividad en la generación de ciencia y tecnología es de 1.000 por año. También nuestras cifras muestras diferencias importantes al ser comparadas con otros países, por ejemplo, Brasil, forma alrededor de 6.000 doctores por año.

No cabe duda que es obvio que si queremos alcanzar el umbral del desarrollo debemos invertir más en la formación de alto nivel en el país; pues de lo contrario no solo estamos limitando la investigación de punta sino que, igualmente, la enseñanza en las más de 60 universidades del país en cuyas aulas menos de la mitad de los docentes cuenta con algún postgrado. De hecho es preocupante que Chile se encuentre por debajo del promedio de los países de la OCDE en cuanto a personal científico y tecnológico, con sólo 12 investigadores por cada 10 mil personas en la fuerza de trabajo, cinco veces menos que el promedio de los países de la OCDE (MINEDUC, 2004). En la actualidad nuestro país muestra tasas de inversión en ciencia y tecnología muy por debajo de aquellos países que hemos consignados como interesantes de emular en sus proceso de transformación socio-educativo y tecnológica. Chile invierte sólo el $0,6 \%$ del Producto Interno Bruto (PIB) en esta área, en cambio países como Finlandia y Corea invierten un $2,7 \%$. Siendo el promedio de los países del OCDE de 2,2\%. (CONYCIT, 2002). 


\section{Apertura a la Innovación Tecnológica y Alfabetización Digital}

En la sociedad de la información surgen nuevas desigualdades tanto o más dramáticas que las producidas en sociedades precedentes; el abismo ahora no es territorial o por escasez de recursos o expoliación, sino que por el acceso a un bien que es inmaterial: el acceso a la información y, en consecuencia, la apertura a la innovación tecnológica. El acceso a ella permite señalar que una sociedad se divida según la cantidad de información que las personas tienen; A esta sociedad dual, Adell (1997) la ha dicotomizado como los "inforicos o infopobres".

Esta revolución tecnológica ha creado redes de comunicaciones globales las que están impactando diariamente los mercados, la cotidianeidad y la cultura, los procesos sociales y políticos. También la democracia se ha visto beneficiada por formas de comunicación global que expanden las alternativas informativas, lo que permite a la gente contar con más posibilidades de saber lo que sucede y, por consiguiente, sistemas rígidos y jerárquicos de control de la información se hacen obsoletos rápidamente.

Pero también, esta revolución global comunicativa va haciendo patente las diferencias entre países para enfrentar educativa y tecnológicamente los efectos de una reestructuración económica, cambios tecnológicos y transformaciones sociales y culturales. Estas diferencias se empiezan a señalar como, por ejemplo, lo que el sociólogo catalán Manuel Castells denomina la "brecha digital", es decir, la idea de que Internet está creando un mundo dividido entre los que tienen y los que no tienen Internet. Tanto a nivel de personas, países y territorios se expresa hoy una gran diferencia de conectividad, aún cuando esta conectividad va en aumento. De acuerdo a cifras del 2002 la participación de América Latina en conectividad a Internet a nivel global alcanzaba sólo al $4 \%$.

Pero lo preocupante va por el lado de la capacidad educativa para ocupar estos medios tecnológicos y aprovecharlos en sus reales posibilidades. Es verdad que el conocimiento está en la red, el conocimiento codificado, pero no el conocimiento que se necesita para lo que se quiere hacer, de lo que se trata es de saber dónde está la información, cómo buscarla, cómo procesarla, cómo transformarla en conocimiento específico para la resolución de problemas (Castells, 1997). Ese es el lugar en donde esta la divisoria digital en estos momentos en el mundo; vale decir, cómo los países se orientan en sus sistemas educativos, tecnológicos y económicos a la sociedad del conocimiento y así, romper con la brecha digital que nos separa con los países que lideran las transformaciones.

Diversos estudios nos señalan que los países desarrollados concentran el 75\% de los accesos a Internet, y con ellos generan el 97\% del comercio electrónico mundial.

La capacidad de absorción de las nuevas tecnologías, y no sólo el acceso a ellas, es clave para cerrar las diferencias introducidas por la Brecha Digital. Si se mide la Brecha Digital a través de la capacidad de absorber TICs, se observa que las distancias entre los países avanzados y las economías en desarrollo son enormes, pero que al menos parecen comenzar, sino a reducirse, al menos a estabilizarse.

El tamaño de la brecha entre los países desarrollados y las economías emergentes es de un 66\% y en 2002 se redujo por primera vez. En el caso de los países más pobres, el tamaño de la brecha es de casi un $90 \%$ y sigue en aumento, aunque a tasas decrecientes.

En un reciente estudio realizado (2003) se entregan interesantes antecedentes en torno al avance desigual que se encuentran en América Latina en torno a la conectividad a Internet. El cuadro siguiente muestra los niveles de penetración de acuerdo al número de usuarios por 100 habitantes: 


\begin{tabular}{|} 
Penetración de Internet (2003) \\
\hline Estados Unidos & 49,95 \\
\hline Japón & 45,47 \\
\hline Chile & 20,02 \\
\hline España & 18,27 \\
\hline Perú & 11,50 \\
\hline Venezuela & 5,28 \\
\hline Brasil & 4,66 \\
\hline México & 3,62 \\
\hline Colombia & 2,70 \\
\hline
\end{tabular}

En el caso de Chile, los resultados de la carera digital son aún limitados. Un estudio realizado en el 2003 por el Centro de Investigación y Desarrollo de la Educación (CIDE) concluyó que el $71 \%$ de los chilenos no tiene los conocimientos necesarios para usar las herramientas que entrega el Internet. Allí se consigna que el $63,2 \%$ de los chilenos no conoce los procesadores de textos y el 67,8 \% no sabe utilizar planillas de cálculo. También el estudio reveló que la población adulta y la de menores ingresos es la que menos maneja las tecnologías y que, al interior del grupo familiar, las mamás son las más rezagadas en el uso del computador $(75,7 \%)$.

Los avances en el plano de la incorporación de TICs al mundo de las organizaciones empresariales tampoco pueden ser considerados altamente efectivos. Un reciente estudio realizado a líderes de opinión en Chile (País Digital, 2003) sobre percepción de la innovación tecnológica concluyó que éstos perciben, en general, que la posición internacional de Chile en desarrollo tecnológico se ha deteriorado durante los últimos años.

\section{Percepción de Posición de Chile en Desarrollo de Tecnología}

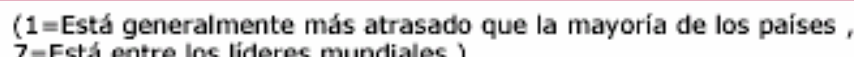

$7=$ Está entre los líderes mundiales )

\section{$\mathrm{N}=345$, Mediana: 3}
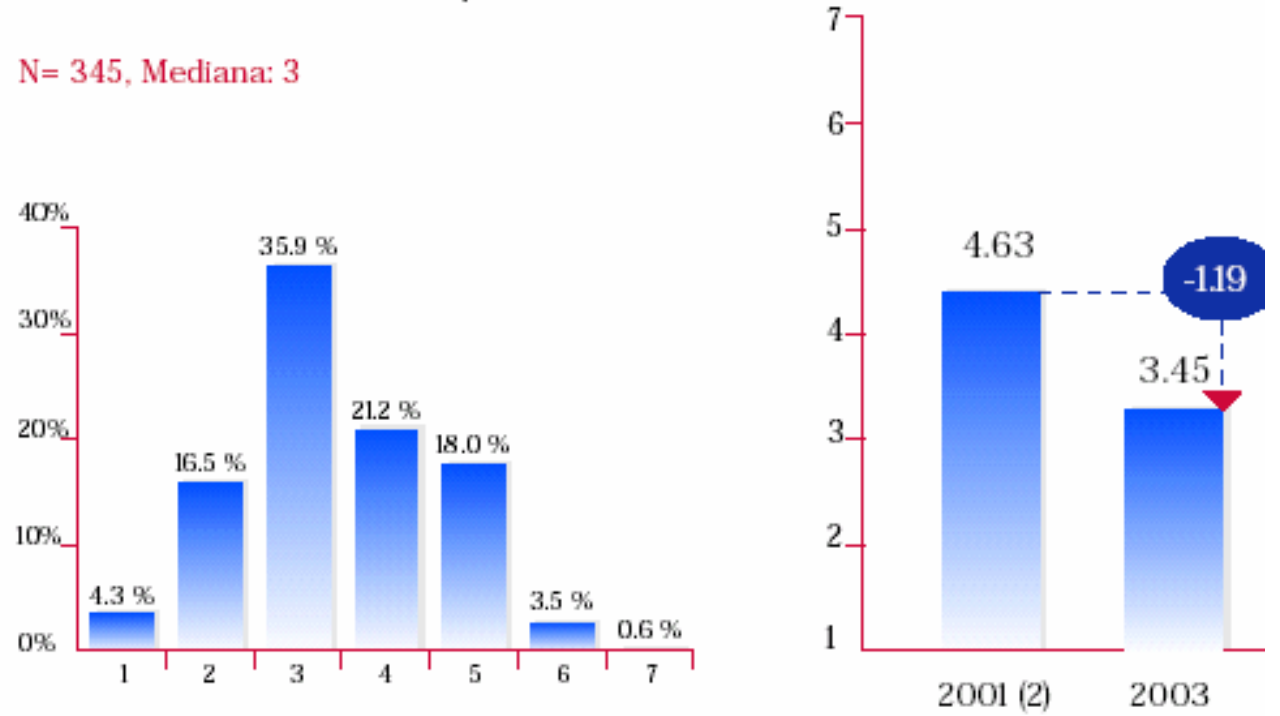

Fuente: Fundación País Digital, Agosto 2003. 
Del mismo modo, se plantea una disminución en la percepción de importancia de las TICs como prioridad para el gobierno y se concluye que la mayor parte de las empresas en las distintas industrias no invierten mayormente en $\mathrm{I}+\mathrm{D}$, y obtienen tecnología por medio de licencias, importación o imitación de empresas extranjeras. Como complemento a lo anterior, existe una percepción de que la colaboración de universidad y empresa en actividades de $\mathrm{I}+\mathrm{D}$ es baja.

El efecto colateral de lo referido anteriormente es que el país posee una baja producción de conocimiento tecnológico, medido a partir de las cifras promedio de patentes concedidas por millón de habitantes: 1,0. En cambio, a Finlandia se le han concedido 119,4, Irlanda 32.4 por millón de habitantes y Corea 70,1 (World Economic Forum, 2002).

La comunidad científica del país viene desde hace un tiempo señalando que una forma pertinente de enfrentar los desafíos tecnológicos y, por cierto, la búsqueda de competitividad del país en el mundo global es contar con un Ministerio de Ciencia, Tecnología e Innovación que permita evitar duplicaciones de instrumentos y recursos públicos y romper con un conjunto de barreras estructurales que limitan la generación de iniciativas de innovación. En la medida que se protejan las patentes intelectuales y se incentive la creación de consorcios tecnológicos encargados de cuidar el conocimiento con valor agregado que producen los actores productivos del país, podremos visualizar rutas claras y efectivas para activar realmente un desarrollo del capital humano avanzado del país, sustentado en un necesario impulso de la alfabetización digital.

\section{Bibliografia}

Adell, J. Redes y educación. En De Pablos, J. y Jiménez, J. (Eds.). 1997. Nuevas Tecnologías, Comunicación Audiovisual y Educación. Barcelona: Ed. Cedecs.

Brunner, J.J. y G. Elacqua. 2003. Informe Capital Humano en Chile, Universidad Adolfo Ibáñez, Escuela de Gobierno. Santiago de Chile.

Castells, Manuel. 1997. La Era de la Información: (vol. 1 La sociedad red; vol. 2 El poder de la identidad; vol. 3 Fin de milenio). Madrid: Alianza Editorial.

Centro de Investigación de las Telecomunicaciones. 2003. Conectividad en América Latina, (Cintel), Colombia.

Fundación País Digital. Encuesta de Percepción a Líderes de Opinión. Agosto, 2003. http://www.paisdigital.org/docs/f117_.pdf

Haefner, Carlos. 2004. Nuevas Tecnologías de Información y Modernización del Sistema Educativo. Revista AULA, año 3, No 5, Departamento de Educación Municipal, Puerto Montt.

Sen, A. 1997. Bienestar, Justicia y Mercado, Barcelona: Paidós. 